\title{
Application of Self-Organizing Map for analyzing robotic arm's action with Consciousness-Based Architecture module
}

\author{
Wisanu Jitviriya \\ Department of Mechanical Information Science and Technology, Kyushu Institute of Technology, \\ 680-4, Kawazu, Iizuka, Fukuoka, 820-8502, Japan \\ E-mail:wisanu@mmcs.mse.kyutech.ac.jp \\ Eiji Hayashi \\ Department of Mechanical Information Science and Technology, Kyushu Institute of Technology, \\ 680-4, Kawazu, Iizuka, Fukuoka, 820-8502, Japan \\ E-mail:haya@mse.kyutech.ac.jp
}

\begin{abstract}
Our research has been focused on developing human-robot interactions, the robots need to perform a high level of intellectual activity and user compatibility. Therefore, we considered the primary structure of a conscious human/animal action, which can be represented by the sequence process, Perception $\rightarrow$ Motivation $\rightarrow$ Action. We have improved a hierarchical structure model, which defines the relationship between the consciousness field and the behavior module. This model is called Consciousness-Based Architecture (CBA). Furthermore, the robot should select the action itself, we have investigated the application of brain-inspired technology so we introduced a SelfOrganizing Map (SOM) neural network that is trained using unsupervised learning to classify its behavior according to the motivation value in the CBA module. In this paper, we attempt to describe the integration of a Self-Organizing Map (SOM) method into a CBA module in order to classify and select autonomous behavior. We confirmed the effectiveness of the proposed system by the experimental results.
\end{abstract}

Keywords: Consciousness-Based Architecture (CBA), Human-robot interactions, Self-Organizing Map (SOM).

\section{Introduction}

Nowadays, the rapid development of high technology has produced non-industrial robots such as robotic pets for kids, home robots, therapeutic robots and service robots. These robots are designed for entertainment, guide in museums, education, welfare and other purposes. The necessary challenge in the development of these robots is a high level of intellectual ability and user compatibility. "User compatibility" in the future robot is an important for ease of use, robot friendliness, human-like capricious behavior and the robots are more attractive to people if their movements are not endlessly repeated. However, so far the development of the robots has met with problems in the recognition system, memorizing the situation, the action selection and communication etc., when the robots interact with human. Therefore, our research has been focused on developing the robot that has process of the actions and consciousness resembling that of a human or an animal which is able to enhance the liveliness and the likelihood of their interactions with users [1]-[3]. We have constructed a hierarchical structure model, which defines the relationship between the consciousness field 
Fig.1. System Structure of Conbe-I
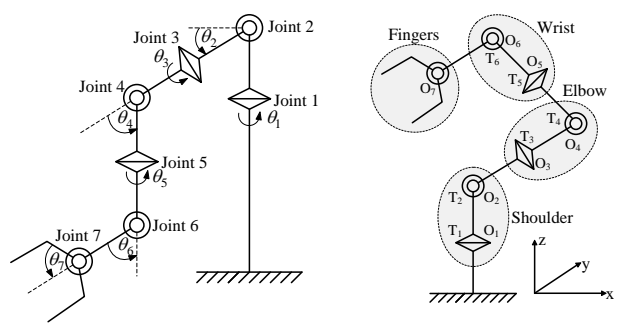

Fig.2. Degree of freedom in the robotic arm and the robotic arm is divided into 4 parts.

and behavior module in the robot. This model is called Consciousness-Based Architecture (CBA). We have improved the integration of a Self-Organizing Map (SOM), one kind of unsupervised artificial neural networks (ANNs) proposed by Kohonen [4]-[6], and based on the neuron organization and decision-making process in the human brain into CBA module. In order to realize a practical robot that can autonomously choose its behavior and adapt to its environment.

In this paper proposes an unsupervised classification method or Self Organizing Map (SOM) combined with the CBA module for use with a robotic arm. First, we showed the outline of the proposed overview system and described an autonomous behavior system of a conscious behavior robot (Conbe-I), consisting of a recognition of the external situation using a web camera, calculating the naturally occurring dopamine and determining the robot's motivation, selecting an action using Self Organizing Map combined with CBA module and the drive of the actuator which accepted an action. Next, we described the SOM context that consists of the classification as "liking" behavior and "disliking" behavior when the robot recognizes the green and blue object, respectively, and the normalization process for preparing the input data to be suitable for training. Finally, the kinds of actions such as look around, interest, be alert, approach, catch the object and avoid were classified using a weight matrix derived by dividing the cells on the map based on unsupervised learning, the robot could select an action by finding the maximum similarity of the each action.

\section{System Structure of Conbe-I}

The figure 1 shows the appearance of a conscious behavior robot (Conbe-I), the robotic arm is divided into 2 parts, an arm and a hand. The arm part has 6 levels of flexibility: the shoulder (Joint1, Joint2), the elbow (Joint3, Joint4) and the wrist (Joint5, Joint6). The hand part has 3 fingers and one level of flexibility. So thus the robotic arm has a total of 7 degrees of freedom as shown in Fig.2 and a small web camera equipped at the palm of the robotic arm which is able to recognize an external situation. The web camera and the multiple actuators are controlled using the RS-485 serial data communication.

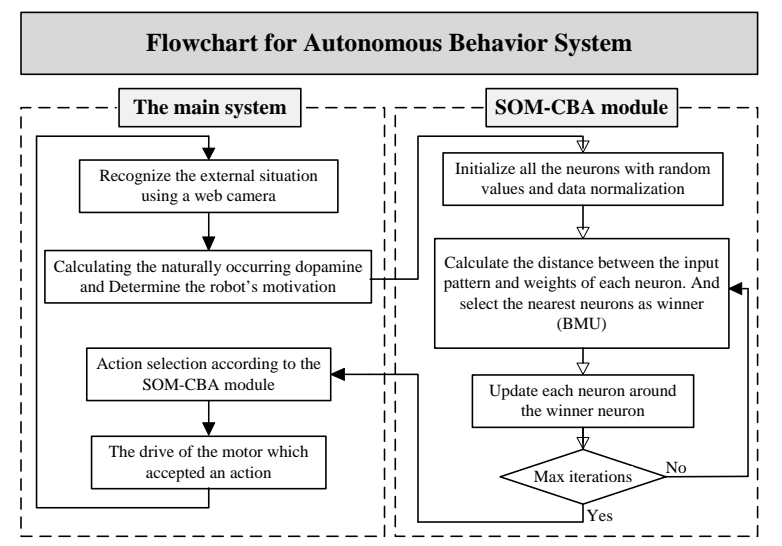

Fig.3. The flowchart of autonomous behavior system

\section{Autonomous Behavior System}

McCarthy has indicated that a robot will need to consider and introspect in order to operate in the common sense world, it will need to have a consciousness and introspective knowledge and some philosophy [7], [8], so we describe the overview system by the flowchart of the autonomous behavior system is shown in Fig. 3 and the details of each item are described below.

\subsection{Recognize an external situation by a web camera}

In this research, the images were simplified by divided into three color groups: green, blue and flesh-color, which were distinguished and recognition in terms of the shape, size and center-of-gravity position as shown 

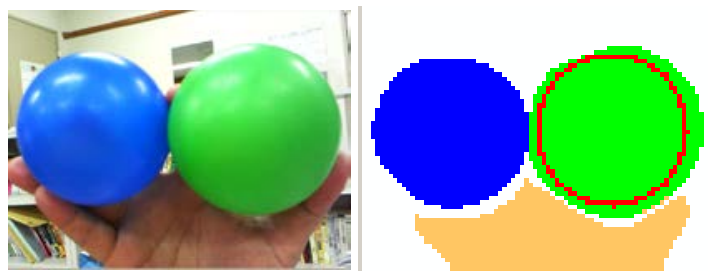

Fig.4. Simplified image.

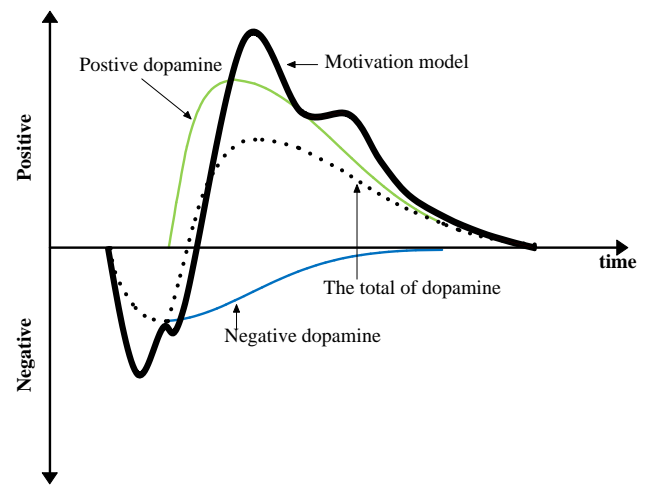

Fig.5. Motivation model.

in Fig.4. The robot could recognize the position and its distance from the target object in order to calculate the naturally occurring dopamine model.

\subsection{Calculating the naturally occurring dopamine and determining the robot's motivation}

When humans and animals take various actions, changes occur in the dopamine in the brain. Dopamine affects brain processes that control movement, emotion response, and ability to experience pleasure and pain, so we used the typical pattern of naturally occurring dopamine for determining the robot's motivation. The waveform of naturally occurring dopamine divided into the rise and fall parts, which are given by Equation (1) and (2) respectively, where $y(t)$ is the naturally occurring dopamine, $x(t)$ is the input data from image recognition, $\omega_{n}$ is the natural angular frequency, $\zeta$ is the damping factor and $T$ is the time constant.

$$
\begin{gathered}
y^{\prime \prime}(t)+2 \zeta \omega_{n} y^{\prime}(t)+\omega_{n}^{2} y(t)-\omega_{n}^{2} x(t)=0 \\
T y^{\prime}(t)+y(t)=x(t)
\end{gathered}
$$

The total of dopamine is calculated by the sum of their positive (the green objects) and negative values (the blue objects), then the total of dopamine is considered as the input variable for determining the robot's motivation as shown in Fig.5 and expressed by a 2nd order system of linear difference equation as shown in

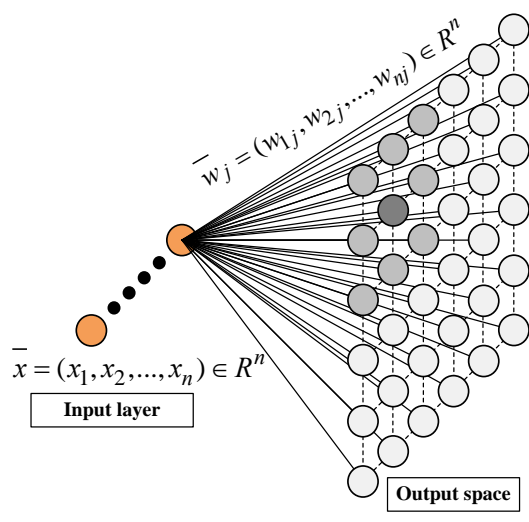

Fig.6. A basic rectangular SOM topology

Equation (3). Here, motivation( $t$ ) is the robot's motivation and $u(t)$ is the total of naturally occurring dopamine.

$$
\begin{aligned}
\text { motivation }^{\prime}(t)+2 \zeta \omega_{n} \text { motivation }^{\prime}(t) \\
\omega_{n}^{2} \text { motivation }(t)=\omega_{n}^{2} u(t)
\end{aligned}
$$

\subsection{Choose an action using a Self-Organizing Map method with CBA module}

In this section, we are aiming for an integrated development the autonomous behavior system that combines a sample the Self-Organizing Map and CBA module in order to classify and select its behavior correspond to its environment and the details are explained below.

\subsubsection{Self-Organizing Map Algorithm}

The basic Self-Organizing Map (SOM) consists of the input layer and the output layer which is fully connected with the input layer by the adjusted weights. After learning of the SOM, the weight vector of a particular cell defines the center of a certain class of the input. In Fig.6 a typical two-dimensional rectangular SOM is shown. The input vector $\bar{x}=\left(x_{1}, x_{2}, \ldots, x_{n}\right) \in R^{n}$ is connected to cell $j$ and the respective connection has a weight $\bar{w}_{j}=\left(w_{1 j}, w_{2 j}, \ldots, w_{n j}\right) \in R^{n}$. The learning of the SOM is performed in the following steps:

Step 1. Initially, we assign to the weight vectors $\bar{w}_{j}(0)$ random values, and we normalize the values of $\bar{w}_{j}(0)$ in $[0,1]$.

Step 2. Present an input vector $\bar{x}$ to the network, then we compute the cell $j$ that is closest to it by means of the Euclidean distance and then find the winner of unit $c$ (Best Matching Unit: BMU) which has the 
minimum Euclidean distance as expressed by Equation (4).

$$
U_{c}=\min \left\|\bar{x}_{j}-\bar{w}_{j}(t)\right\|
$$

Step 3. The adaptation process adjusts the weight vector of cell $j$ and the weight vectors of its neighboring cells by Equation (5).

$$
\bar{w}_{j}(t+1)=\bar{w}_{j}(t)+\eta(t) \cdot\left[\bar{x}(t)-\bar{w}_{j}(t)\right]
$$

Step 4. The time $t$ increases to $t+1$, if $t<T$ then go to step 2; otherwise stop the training.

Where, it is the time of the iteration, $T$ is the predefined number of iterations and $\eta(t)$ is the learning rate, which is decreasing function of time by Equation (6).

$$
\eta(t)=\eta_{0} \cdot \exp (-t / T)
$$

\subsubsection{Combine the SOM with CBA module}

Our previous research, the CBA model decides on the conscious level that the robot must strongly consider and select the behavior corresponding to the conscious level. In this paper, the CBA has been developed as shown in Fig.7, the CBA model has a 3-level hierarchy in the motivation module that connects with a SOM module for clustering actions in a 3-level hierarchy in the behavior module.

\section{Experimental results}

\subsection{Classification results}

The actions of the robotic arm are divided into two characteristic behaviors, according to the value of the motivation and the effect of surface color on the object recognition. For instance, when the robot sees a green object (Liking behavior), the motivation value rises and the robot looks around and interests the target object after that the robot attempts to approach or catch the object as shown in Fig.8. On the other hand, when the robot recognizes a blue object (Disliking behavior), the motivation value drops and the robot should be alert and avoid the object as shown in Fig.9. In addition, when the robot recognized the flesh color, the actions are clustered around interested behavior and alert behavior. In this paper, the Min-Max Normalization technique is used to scale the input sample in the range of 0 and 1 depending on its relative size. The parameters of SOM as shown in Table 1.

To make it easy to understand, Fig.10 shown the all actions of the robotic arm, which were classified using a

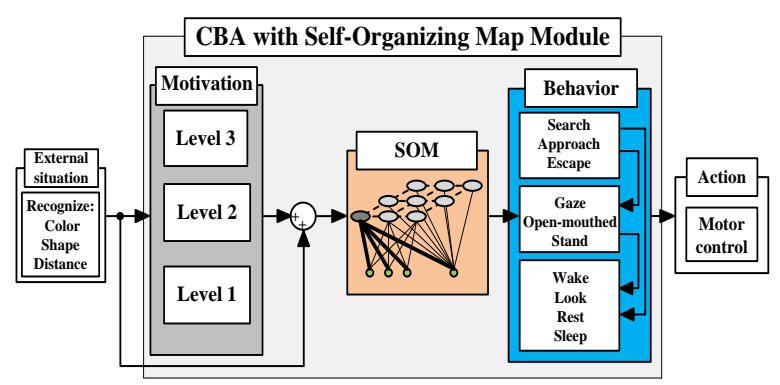

Fig.7. Combine SOM into CBA module.
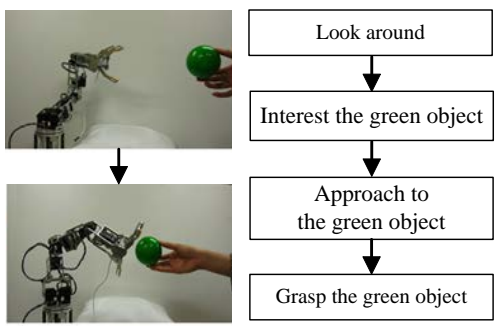

Fig.8 “Liking behavior”: recognize the green object

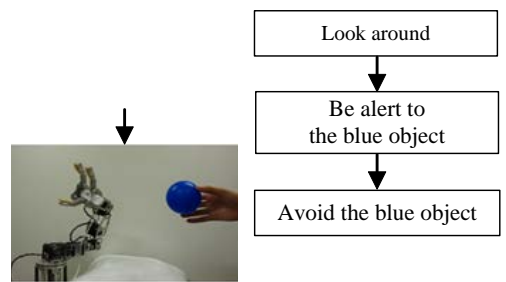

Fig.9 "Disliking behavior": recognize the blue object

Table 1. The parameters of SOM.

\begin{tabular}{|l|c|l|}
\hline & Value & \multicolumn{1}{c|}{ Description } \\
\hline Moti_flag & 0 and 1 & $\begin{array}{l}\text { The positive value is } 1 \text {, and } \\
\text { the negative value is } 0 .\end{array}$ \\
\hline Moti_value & 0 to 1 & The motivation value \\
\hline Green & 0 to 1 & Pixel of green color \\
\hline Blue & 0 to 1 & Pixel of blue color \\
\hline Flesh & 0 to 1 & Pixel of flesh color \\
\hline$t$ & 1000 & The number of learning \\
\hline$\eta_{0}$ & 0.35 & The initial learning rate \\
\hline Map size & $20 \times 20$ & $\begin{array}{l}\text { The rectangular SOM } \\
\text { topology size }\end{array}$ \\
\hline
\end{tabular}

weight matrix derived by dividing the cells on the map based on unsupervised learning SOM. For instance, if the input data was Moti_flag $=1$, Moti_value $=0.5$, Green $=0.8$, Blue $=0$ and Flesh $=0.1404$. The robot recognized the green object and the target object was near the robot's hand, when the system finished the SOM learning. The blue cells were shown in Fig.10, which were the winner node of each behavior and the 
green cell was the response action from SOM learning, so in this state the robot autonomously selected the "Catch the object" action.

\subsection{Verification experiment}

We let the robotic arm recognized a green ball (Liking) and a blue ball (Disliking). We observed the action of the Conbe-I until it took a green ball from the human's hand. The transition of the motivation is shown in Fig.11. After the Conbe-I was started it recognized the green ball at $\mathrm{T} 1$, then the value of motivation increased steadily and the robot selected the action between looking around behavior and an interesting behavior. From T2-T3, it was selected “Approach” action and it began to approach the green ball and opened the fingers after that the robot came close enough to the green ball to catch it in a time interval (T3-T4), but on the other hand, the blue ball was shown in order to decrease the robot's motivation and the motivation became to the negative value. In the time interval from $\mathrm{T} 5$ to $\mathrm{T} 8$ that shown the disliking behavior that consists of "Look around”, “Alertness” and “Avoidance” respectively.

\section{Conclusion}

In this paper, we present the sample application of Self Organizing Map (SOM) for analyzing and clustering the robotic arm's action. From the experimental results, 9 motions of the robotic arm could be classified on the map of SOM and the robotic arm could autonomously select its behavior and adapt to its surrounding environment. In the future study, our research will continue to develop the system of Conbe-I such as it becomes possible to create an emotional system and apply the memorization system based on an artificial neural network into the robot's system.

\section{References}

1. N. Goto and E. Hayashi, Design of Robotic Behavior that imitates animal consciousness (Journal of Artificial Life and Robotics Vol.12, Springer 2008 ), pps. 97-101.

2. Eiji Hayashi, Takahiro Yamasaki and Koichiro Kuroki, Autonomous Behavior System Combing Motivation with Consciousness Using Dopamine (IEEE International Symposium on Computational Intelligence in Robotics and Automation, 2009), pp.126-131.

3. Motoki Yoshida, Eiji Hayashi, Design of robotic behavior that imitates animal consciousness: Construction of the user-recognition systems (Artificial Life Robotics, AROB(17), 2012).

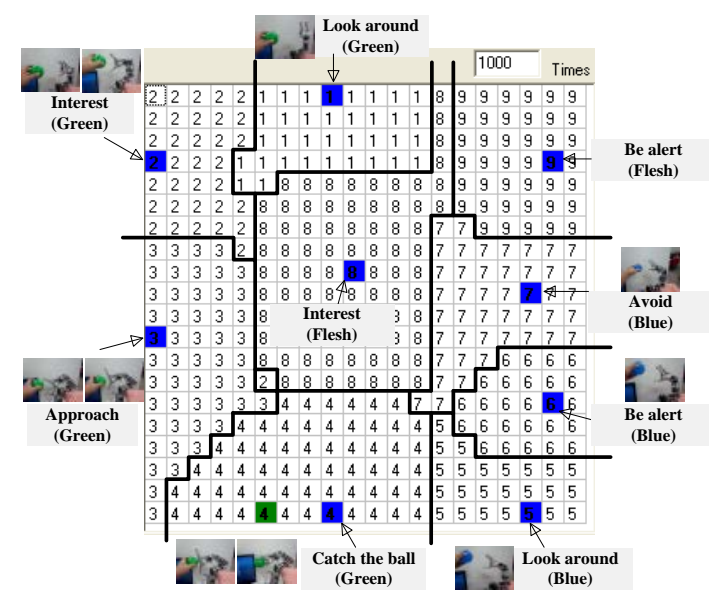

Fig.10. The classification on the map.

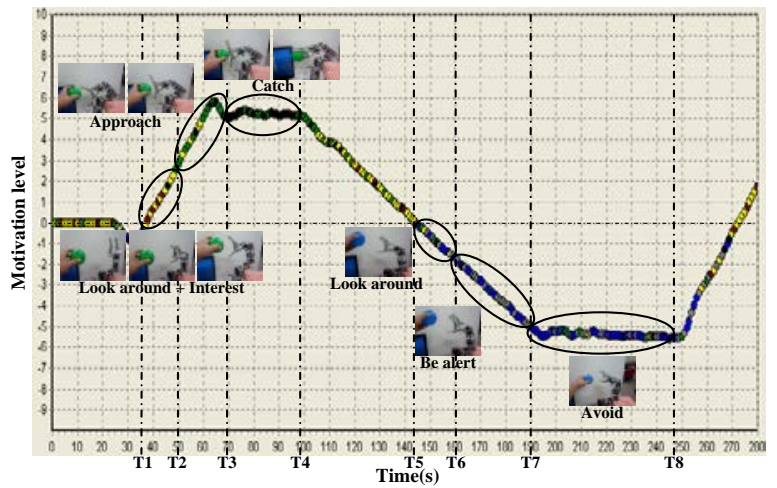

Fig.11. Transition of the motivation.

4. T. Kohonen, Self-Organizing Maps (Series in Information Sciences, Vol. 30, Springer, Heidelberg. Seconded, 1995).

5. T. Kohonen, The self-organizing map (Neurocomputing, Vol.21, 1998), pp.1-6.

6. T. Kohonen, Essentials of the self-organizing map (Neural Networks, Vol. 37, 2013), pp.52-65.

7. McCarthy J (1995) Proceedings of the fourteenth international joint conference on Artificial Intelligence, IJCAI 95, pp.2041-2044.

8. McCarthy J (1996) Making robots conscious of their mental states (In: Muggleton S (ed) Machine intelligence, vol. 15, Oxford University Press, Oxford), pp. 3-17. 